Pacific Journal of Mathematics

HOMOTOPICALLY TRIVIAL TOPOSES MARVIN Victor Milk 


\title{
HOMOTOPICALLY TRIVIAL TOPOSES
}

\author{
M. V. MIELKE
}

We give a number of equivalent conditions for a topos to be homotopically trivial and then relate these conditions to the logic of the topos. This is accomplished by constructing a family of intervals that can detect complemented, regular subobjects of the terminals. It follows that these conditions generally are weaker than the Stone condition but are equivalent to it if they hold locally. As a consequence we obtain an extension of Johnstone's list of conditions equivalent to DeMorgan's law. Thus, for example, the fact that there is no nontrivial homotopy theory in the category of sets is equivalent to the fact, among others, that maximal ideals in commutative rings are prime. Moreover, any topos has a 'best approximation' by a locally homotopically trivial topos.

1. Homotopy in a topos. The notion of (singular) homotopy in a topos is the notion of homotopy based, as in the topological case, on an interval, where by an interval $I$ in a topos $E$ is meant an internally linearly ordered object of $E$ with disjoint minimum $m: 1 \rightarrow I$ and maximum $M$ : $1 \rightarrow I$ elements, i.e., $m \cap M=0$. More precisely, for an interval $I$ in $E$, an (ordered) pair of maps $f, g: A \rightarrow B$ in $E$ is said to be directly I-homotopic (abbreviated DI-homotopic) if there is a map $h: A \times I \rightarrow B$ such that $f=h($ id $\times m)$ and $g=h($ id $\times M): A \simeq A \times 1 \rightarrow A \times I \rightarrow B$, and to be I-homotopic if there is a finite sequence $\left\{j_{k}\right\}, k=1, \ldots, n+1$, of maps $A \rightarrow B$ with $j_{1}=f, j_{n+1}=g$ and $j_{k} D I$-homotopic to $j_{k+1}$ or vice versa, for $k=1, \ldots, n$. $E$ is said to be $(D) I$-homotopically trivial if every pair of parallel maps in $E$ are ( $D) I$-homotopic. It is readily seen that $E$ is both $D I$ - and $I$-homotopically trivial for any interval $I$ that is trivial in the sense that $I \simeq I_{1} \amalg I_{2}$ and $m, M$ factor through $I_{1}, I_{2}$ respectively. In fact we have:

1.1 Proposition. For an interval $I$ in a topos $E$, the following conditions are equivalent:

(1) I is trivial.

(2) $E$ is DI-homotopically trivial.

(3) $E$ is I-homotopically trivial.

We postpone the proof that $(3) \Rightarrow(1)$ until 2.2. Note that if $I$ is trivial then $D I$-homotopy is both symmetric and transitive but the converse need 
not hold. For example the "topological topos" of [5] contains the standard unit internal $I=[0,1]$ which is not trivial but $D I$-homotopy is symmetric and transitive. However, the converse does hold for intervals that are irreducible in the sense that $\neg m=M$ and $\neg M=m$. We have:

1.2 Proposition. For an irreducible interval $I$ in a topos $E$, the following conditions are equivalent:

(1) I is trivial.

(2) DI-homotopy is symmetric.

(3) DI-homotopy is transitive.

We give the proof in 3.2 and 4.1. It is clear, from 1.1, that $E$ is homotopically trivial (i.e., all intervals in $E$ are trivial) then $D$-homotopy (i.e., $D I$-homotopy, for all intervals $I$ in $E$ ) is both symmetric and transitive, in fact:

1.3 Proposition. For a topos E, the following conditions are equivalent:

(1) E is homotopically trivial.

(2) D-homotopy is symmetric.

(3) D-homotopy is transitive.

The proof depends on, and immediately follows, 4.3.

So far we have considered conditions equivalent to homotopy triviality. We now turn to a somewhat weaker condition. Recall that a Heyting algebra $H$ is called a Stone lattice if the regular (i.e., the $\neg \neg$-closed) elements of $H$ have complements, or, equivalently, if the equation $\neg x \vee$ $\neg \neg x=t$ holds for all $x \in H$. (See [3] or problem 3, p. 162, [2].)

1.4 Proposition. For the following conditions on a topos $E$ : (1) E is homotopically trivial; (2) the Heyting algebra of subobjects of 1 is a Stone lattice; (3) all regular subobjects in $E$ have complements, the following implications hold: (a) (1) implies (2), (b) (3) implies (1), and (c) if E satisfies $S G$ (subobjects of 1 generate E), (2) implies (3).

The proof depends on, and follows, 5.1.

We can now state and prove the main result which is a strengthened form (without the $S G$ condition) of 1.4 obtained by passing to local notions. A topos $E$ is said to be locally homotopically trivial if the topos $E / X$ is homotopically trivial for all $X \in E$. 
1.5 THEOREM. For a topos $E$, the following conditions are equivalent.

(1) $E$ is locally homotopically trivial.

(2) Regular subobjects in E have complements.

(3) $E$ is a Stone topos (i.e., the subobject classifier $\Omega$ is an internal Stone lattice).

(4) DeMorgan's law $(\neg(p \wedge q) \Leftrightarrow \neg p \vee \neg q)$ holds in $E$.

Proof. Condition (1) implies, for $X \in E$, that $E / X$ is homotopically trivial and thus, by $1.4(\mathrm{a})$, that the regular subobjects of 1 in $E / X$ have complements. Since subobjects of 1 in $E / X$ correspond bijectively to subobjects of $X$ in $E$ and since this correspondence respects regularity and complements, it readily follows that (1) implies (2). Conversely, if (2) holds then regular subobjects of 1 in $E / X$ have complements, for all $X \in E$. Since regular subobjects of $(f: Y \rightarrow X) \in E / X$ correspond to regular subobjects of 1 in $((E / X) / f) \simeq E / Y$, it follows that regular subobjects of $E / X$ have complements and consequently, by 1.4(b), (2) implies (1). The equivalence of (2), (3), and (4) is essentially Theorem 3 of Frink [1]. Also see [3].

There are many equivalent forms of 1.5(3) (see [3]) and of 1.5(4) (see [4]) and thus of 1.5(1). We list a few:

1.6 COROLlary. For a topos $E$, the following conditions are equivalent:

(1) $E$ is locally homotopically trivial.

(2) In commutative, unitary rings in $E$, maximal ideals are prime.

(3) If $E$ has a natural number object, the Dedeking reals are conditionally order-complete.

(4) (If $E=\operatorname{Shv}(X)) X$ is extremally disconnected.

(5) (If $E=(\text { Set })^{C \text { op }}$ ) for every pair $f, g$ of maps of $C$ with common codomain there are maps $x, y$ such that $f x=g y$.

There are also many conditions which imply $2.5(1)$.

1.7 CoROllary. Each of the following conditions on a topos $E$ implies that it is locally homotopically trivial.

(1) E satisfies the axiom of choice.

(2) $E$ is Boolean $(\Omega \simeq[2])$.

(3) $E$ is linear (i.e., $\Omega$ is an interval in $E$ ).

Proof. By 5.23 [2], (1) implies (2), clearly (2) implies (3), and (3) implies 1.5(3) by Theorem 3 [3]. 
Finally, in view of $1.4(\mathrm{c})$, we have:

1.8 Corollary. If $E$ satisfies $S G$ then the conditions of $1.3,1.5$, and 1.6 are equivalent and all are implied by each condition of 1.7 .

1.9. REMARK. By 1.5 constructions and results involving DeMorgan toposes can equally well be interpreted in terms of locally homotopically trivial toposes. For example, the results of [6] may be viewed as showing that any topos has a 'best approximation' by a locally homotopically trivial topos, while those of [7] characterize the locally homotopically trivial toposes, among the Set-toposes satisfying $S G$, as the projective toposes.

We end this section with an example of a topos that is homotopically trivial but not locally homotopically trivial. The topos $E=(\mathrm{Set})^{A^{\mathrm{op}}}$ of $A$-sets, where $A$ is the monoid on two generators $a, b$ with $a^{2}=\mathrm{id}$ is homotopically trivial. To see this, note that an interval in $E$ consists of an interval $(I, m, M)$ in Set together with two order and endpoint preserving maps $a, b: I \rightarrow I$ with $a^{2}=$ id. However, if $a(x) \leq x$ then $x=a^{2}(x) \leq$ $a(x)$, thus, by comparability and antisymmetry, $a=\mathrm{id}$. From this it readily follows that $I=I_{1} \amalg I_{2}$, where $I_{1}=\left\{x \mid b^{n}(x)=m\right.$, for some integer $n \geq 0\}$ and $I_{2}=I-I_{1}$, renders $I$ trivial in $E$. We now lift the interval $\pi: 2 \times[3] \rightarrow 2$ in Set $/ 2$, where $2=\{0,1\},[3]$ is the ordinal $\{0<1<2\}$ and $\pi$ is the projection on the first factor, to a nontrivial interval $p: I \rightarrow X$ in $E / X$, where $X$ is 2 with $A$-action given by $a(i)=1-i$, $b(i)=0, i \in 2, I$ is $2 \times[3]$ with $A$-action given by $a(i, j)=(1-i, j)$, $b(i, j)=(0,0)$ or $(0,2)$ as $j \leq i$ or $j>i, i \in 2, j \in[3]$, and $p=\pi$. It is easily seen that $p$ is an interval in $E / X$ for which there is no separation of $m=\{(0,0),(1,0)\}$ and $M=\{(0,2),(1,2)\}$ since $b(1,1) \in m$ while $b a(1,1) \in M$.

2. Separation. In this section we complete the proof of 1.1 . We begin with some basic properties of the separation relation $s$ on the set of subobjects of an object of $E$ (for subobjects $A, B$ of $X, A$ s $B$ iff $A$ and $B$ can be separated, i.e., $X \simeq X_{1} \amalg X_{2}$ and $A \leq X_{1}, B \leq X_{2}$ ).

2.1 LeMMA. Let $\mathrm{s}$ denote the separation relation on the set of subobjects of $X \in E$.

(1) $\mathrm{s}$ is symmetric.

(2) 0 s $A$ for all $A \leq X$.

(3) If $A \leq A_{1}$ and $B \leq B_{1}$, then $A$ s $B$ if $A_{1}$ s $B_{1}$. 
(4) $A \mathrm{~s}\left(B_{1} \cup B_{2}\right)$ iff $A \mathrm{~s} B_{1}$ and $A \mathrm{~s} B_{2}$.

(5) If $A=\cup_{i=1}^{n} A_{i}, B=\cup_{j=1}^{m} B_{j}$ then $A \mathrm{~s} B$ if, for each pair $i, j$, there is a pair $A_{i}^{j}, B_{j}^{i}$ such that $A_{i} \leq A_{i}^{j}, B_{j} \leq B_{j}^{i}$, and $A_{i}^{j} \mathrm{~s} B_{j}^{i}$.

Proof. (1), (2) and (3) are trivial and (3) implies the only if part of (4). For the converse suppose $A \leq C_{0}, B_{1} \leq C_{1}, A \leq C_{0}^{\prime}, B_{2} \leq C_{1}^{\prime}$ and $C_{0} \amalg C_{1}$ $\simeq X \simeq C_{0}^{\prime} \amalg C_{1}^{\prime}$. Then

$$
X=\left(C_{0} \cup C_{1}\right) \cap\left(C_{0}^{\prime} \cup C_{1}^{\prime}\right)=C_{0}^{\prime \prime} \cup C_{1}^{\prime \prime},
$$

where $C_{0}^{\prime \prime}=C_{0} \cap C_{0}^{\prime}$ and

$$
\begin{aligned}
C_{1}^{\prime \prime} & =\left(C_{0} \cap C_{1}^{\prime}\right) \cup\left(C_{1} \cap C_{0}^{\prime}\right) \cup\left(C_{1} \cap C_{1}^{\prime}\right) \\
& =C_{1} \cup\left(C_{0} \cap C_{1}^{\prime}\right)=\left(C_{1} \cap C_{0}^{\prime}\right) \cup C_{1}^{\prime} .
\end{aligned}
$$

Clearly $A \leq C_{0}^{\prime \prime}, B_{1} \cup B_{2} \leq C_{1}^{\prime \prime}$, and $C_{0}^{\prime \prime} \cap C_{1}^{\prime \prime}=0$, i.e., $A$ s $\left(B_{1} \cup B_{2}\right)$. Multiple applications of (1), (3) and (4) give (5).

Before completing the proof of 1.1 , note that if $i^{p}: 1 \rightarrow 1 \amalg 1=[2]$, $p=0,1$, are the canonical injections then, for any interval $I$ and map $h$ : $I \rightarrow[2]$, we have, as subobjects of $I$,

$$
(h m)^{*}\left(i^{p}\right)=m^{*} h^{*}\left(i^{p}\right)=m \cap h^{*}\left(i^{p}\right) \leq h^{*}\left(i^{p}\right)
$$

and

$$
(h M) *\left(i^{p}\right) \leq h^{*}\left(i^{p}\right),
$$

where, in general, $f^{*}$ denotes the pullback along $f$. Since coproducts are universal in a topos, $h^{*}\left(i^{p}\right) \amalg h^{*}\left(i^{1-p}\right) \simeq I$ and consequently (i) $(h m)^{*}\left(i^{p}\right)$ $\mathrm{s}(h M)^{*}\left(i^{1-p}\right)$, and (ii) $(h m)^{*}\left(i^{p}\right) \mathrm{s}(h m)^{*}\left(i^{1-p}\right), p=0,1$.

2.2 Proof that (3) implies (1) in 1.1. If $E$ is homotopically trivial then the maps $i^{p}: 1 \rightarrow[2], p=0,1$ are $I$-homotopic and thus there is a sequence $\left\{j_{k}\right\}, k=1, \ldots, n+1$, of maps $1 \rightarrow[2]$, with $j_{1}=i^{0}, j_{n+1}=i^{1}$, and a sequence of homotopies $h_{k}$ rendering $j_{k} D I$-homotopic to $j_{k+1}$ or vice versa, $k=1, \ldots, n$. Since

$$
\begin{gathered}
I=\bigcap_{k=1}^{n}\left(h_{k}^{*}\left(i^{0}\right) \cup h_{k}^{*}\left(i^{1}\right)\right)=\bigcup_{\left(p_{1}, \ldots, p_{n}\right)}^{\bigcup}\left(h_{1}^{*}\left(i^{p_{1}}\right) \cap \cdots \cap h_{n}^{*}\left(i^{p_{n}}\right)\right), \\
m=m \cap I=\bigcup_{\left(p_{1}, \ldots, p_{n}\right)}^{\bigcup} m_{1}^{p_{1}} \cap \cdots \cap m_{n}^{p_{n}}
\end{gathered}
$$

and

$$
M=\bigcup_{\left(q_{1}, \ldots, q_{n}\right)} M_{1}^{q_{1}} \cap \cdots \cap M_{n}^{q_{n}}
$$


where $p_{i}, q_{l} \in\{0,1\}, i=1, \ldots, n$, and

$$
m_{k}^{p_{k}}=m \cap h_{k}^{*}\left(i^{p_{k}}\right)=\left(h_{k} m\right)^{*}\left(i^{p_{k}}\right) \quad \text { and } \quad M_{k}^{q_{k}}=\left(h_{k} M\right)^{*}\left(i^{q_{k}}\right) .
$$

By 2.1(5), $m \mathrm{~s} M$ if for each pair $\left(p_{1}, \ldots, p_{n} ; q_{1}, \ldots, q_{n}\right)$ of tuples there are subscripts $i, j$ with $m_{i}^{p_{l}} \mathbf{S} M_{j}^{q_{s}}$ (we call a pair of tuples with this property a separated pair). We shall show that all pairs are separated by inductively (on $k$ ) analyzing the list of pairs that, possibly, are not separated. Now, by (i), $m_{1}^{p} s M_{1}^{1-p}, p=0,1$, and thus every pair with $q_{1}=1-p_{1}, p_{1}=0,1$, is separated. Since $j_{1}=i^{0}, h_{1} m=i^{0}$ or $h_{1} M=i^{0}$ and thus $m_{1}^{1}=0$ or $M_{1}^{1}=0$. By 2.1(2), then, $m_{1}^{1} \mathrm{~s} M_{1}^{1}$ and every pair with $p_{1}=q_{1}=1$ is separated. Thus, after considering $j_{1}$ and $h_{1}$, we see that the only possible pairs not separated satisfy $p_{1}=q_{1}=0$. Assume, now, that after considering $j_{1}, \ldots, j_{k}$ the only possible pairs not separated satisfy $p_{1}=q_{1}=0$, $i=1, \ldots, k$. Again, from (i), we have that every pair with $q_{k+1}=1-p_{k+1}$, $p_{k+1}=0,1$, is separated. Thus the only possible pairs not separated satisfy $p_{i}=q_{1}=0, i=1, \ldots, k, p_{k+1}=q_{k+1}$. However, since one of the following equations must hold $-h_{k} m=h_{k+1} m, h_{k} m=h_{k+1} M, h_{k+1} m$ $=h_{k} M, h_{k+1} M=h_{k} M$ - it follows from (i) and (ii) that one of the following cases holds: $\left(m_{k}^{0}=m_{k+1}^{0}\right)$ s $M_{k+1}^{1},\left(m_{k}^{0}=M_{k+1}^{0}\right)$ s $M_{k+1}^{1}$, $\left(m_{k+1}^{1}=M_{k}^{1}\right)$ s $M_{k}^{0}, m_{k+1}^{1} \mathrm{~s}\left(M_{k+1}^{0}=M_{k}^{0}\right)$ and since each of these cases separates pairs satisfying $p_{k}=q_{k}=0, p_{k+1}=q_{k+1}=1$, the only possible pairs not separated satisfy $p_{l}=q_{i}=0, i=1, \ldots, k+1$. By induction, then, the only possible pair not separated is $p_{i}=q_{i}=0, i=1, \ldots, n$. But by 2.1(2), $m_{n}^{0} \mathrm{~s} M_{n}^{0}$ since $j_{n+1}=i^{1}$ and, thus, either $h_{n} M=i^{1}$ or $h_{n} M=i^{1}$ and, consequently, either $m_{n}^{0}=0$ or $M_{n}^{0}=0$. Thus all pairs are separated and (3) implies (1).

3. Quotient objects. In this section we note some basic properties of certain quotient objects and prove part of 1.2.

For any object $I$ in $E$, if $C \leq I$ then $R=(C \times C) \cup \Delta(\Delta$ is the diagonal of $I$ ) is readily checked to be an equivalence relation on $I$, and, as such (equivalence relations are effective in a topos, p. 27 [2]) the corresponding coequalizer $R \Rightarrow I \stackrel{q}{\rightarrow} I / C$ has the property that for any pair $f, g: A \rightarrow I, q f=q g$ iff $(f, g): A \rightarrow I \times I$ factors through $R$ iff there is an epi $\beta=\beta_{1}+\beta_{2}: B_{1} \amalg B_{2} \rightarrow A$ such that $f \beta_{1}$ and $g \beta_{1}$ each factors through $C$ and $f \beta_{2}=g \beta_{2}$. Moreover, it is not difficult to show that the $q$-images $q(A), q(B)$ of disjoint subobjects $A, B$ of $I$ are disjoint if $C \leq A$; in fact $B \simeq q(B), q^{*} q(B) \simeq B$, and $q(C)$ is a subobject of 1 .

3.1 LEMMA. If $C$ is a subobject of an object $I$ in a topos $E$ then the image $q(C)$ of $C$ under the coequalizer $q: I \rightarrow I / C$ satisfies

(a) $\neg q(C)=q(\neg C) \simeq \neg C$. 
(b) $q^{*}(q(C)) \simeq C$.

(c) The objects $q(C)$ and $|C|$ are isomorphic, where $|C|$ is the support of $C$, i.e., the image of $C \rightarrow 1$.

(d) If, in addition, $A \cap C=0$, for $A \leq I$, then $\neg q(A)=q(\neg A)$.

Proof. It is easily seen that $\neg q(B) \leq q(\neg B)$ for any $B \leq I$ and for any epi $q$ with domain $I$. But, as noted above, $\neg C \simeq q(\neg C), q(C) \cap$ $q(\neg C)=0=q(A) \cap q(\neg A)$ since $C \cap \neg C=0=A \cap \neg A$ and, if $A \cap$ $C=0, C \leq \neg A$. Thus $q(\neg C) \leq \neg q(C), q(\neg A) \leq \neg q(A)$ and (a) and (d) follow. (b) Since the commutative square $(C \stackrel{i}{\rightarrow} I \rightarrow I / C)=(C \rightarrow q(C) \rightarrow$ $I / C$ ) is readily seen to be a pushout with $i$ mono, it is, by 1.28 [2], also a pullback. (c) follows from the above observation that $q(C)$ is a subobject of 1 .

We can now give:

3.2 Proof that $(1) \Leftrightarrow(3)$ in 1.2. Let $q: I \amalg I \rightarrow J$ be the coequalizer determined, as above, by the subobject $m \amalg M: C=1 \amalg 1 \rightarrow I \amalg I$ of $I \amalg I$. If $i^{p}: I \rightarrow I \amalg I, p=0,1$, are the canonical injections then the maps $q i^{p}$ : $I \rightarrow J$ render $m_{p}=q i^{p} m D I$-homotopic to $M_{p}=q i^{p} M, p=0$, 1. Since $M_{0}=m_{1}$, condition 1.2(3) gives a map $h: I \rightarrow J$ with $h m=m_{0}$ and $h M=M_{1}$. But then, in view of 3.1(b),

$$
\begin{aligned}
m \cap h^{*}(q(C)) & =(h m)^{*}(q(C))=\left(q i^{0} m\right)^{*}(q(C))=m^{*}\left(i^{0}\right)^{*} q^{*}(q(C)) \\
& =m^{*}\left(i^{0}\right)^{*}(C)=m^{*}(M)=m \cap M=0 .
\end{aligned}
$$

Hence $h^{*}(q(C)) \leq \neg m$ and similarly $h^{*}(q(C)) \leq \neg M$. Thus if $I$ is irreducible then $h^{*}(q(C))=0$ and consequently $h$ factors through $\neg q(C)$. Thus, in view of 3.1 (a), $h$ induces a map

$$
I \rightarrow \neg q(C)=\neg C=\neg M \amalg \neg m=m \amalg M \simeq 1 \amalg 1
$$

that separates $m$ and $M$ since $h m=m_{0}$ and $h M=M_{1}$. Since (1) $\Rightarrow(3)$ is trivial, (1) $\Leftrightarrow(3)$.

4. Quotient intervals. In this section we make a detailed study of intervals, culminating in results which lead to proofs of 1.2 and 1.3. We begin with a precise description of an interval: an interval in a topos $E$ consists of an object $I$ of $E$ together with a linear order $L \leq I \times I$ on $I$ ( $L$ is reflexive, transitive, antisymmetric $\left(L \cap L^{-1}=\Delta\right.$, where $L^{-1}$ is the opposite relation to $L$ and $\Delta$ is the diagonal of $I$ ) and comparable $\left.\left(L \cup L^{-1}=I \times I\right)\right)$ together with a minimum $m: 1 \rightarrow I(m \times$ id: $1 \times I$ $\rightarrow I \times I$ factors through $L$ ) and a maximum $M: 1 \rightarrow I$ (id $\times M$ factors 
through $L$ ) element with $m \cap M=0$. In terms of generalized elements (p. 157 [2]) a relation $L$ on $I$ is linear iff the relation $\leq$ induced by $L$ on each set $E(A, I), A \in E$ (for $f, g: A \rightarrow I, f \leq g$ iff $(f, g): A \rightarrow I \times I$ factors through $L$ ) is reflexive, transitive, antisymmetric and for each pair $f, g$ : $A \rightarrow I$, there is an epi $\alpha=\alpha_{1}+\alpha_{2}: A_{1} I A_{2} \rightarrow A$ such that $f \alpha_{1} \leq g \alpha_{1}$ and $g \alpha_{2} \leq f \alpha_{2}$.

We can now give:

4.1 Proof that (1) $\Leftrightarrow(2)$ in 1.2. The nontrivial part is $(2) \Rightarrow(1)$. If $D I$-homotopy is symmetric then, since the identity id: $I \rightarrow I$ renders $m$ $D I$-homotopic to $M$, there is a map $h: I \rightarrow I$ with $h m=M$ and $h M=m$. By comparability there is an epi $\alpha=\alpha_{1}+\alpha_{2}: A_{1} \amalg A_{2} \rightarrow I$ with $\alpha_{1} \leq h \alpha_{1}$ and $h \alpha_{2} \leq \alpha_{2}$. Hence the images $\alpha_{l} A_{1} \leq I$ satisfy $\alpha_{1} A_{1} \cup \alpha_{2} A_{2}=I$ and, by antisymmetry, $\alpha_{1} A_{1} \cap \alpha_{2} A_{2} \leq H$, where $j: H \rightarrow I$ is the equalizer of $h$ and id. But then

$$
H \cap M=j^{*}(M)=(h j)^{*}(M)=j^{*} h^{*}(M)=H \cap h^{*}(M) .
$$

Hence

$$
\begin{aligned}
M \cap H & \leq M \cap h^{*}(M)=M^{*} h^{*}(M)=(h M)^{*}(M) \\
& =m^{*}(M)=m \cap M=0 .
\end{aligned}
$$

Similarly, $m \cap H=0$ and consequently $H \leq \neg M \cap \neg m$. Thus if $I$ is irreducible then

$$
\alpha_{1} A_{1} \cap \alpha_{2} A_{2} \leq H \leq m \cap M=0
$$

and, since $m \leq h M(h M \leq M), m(M)$ factors through $\alpha_{1} A_{1}\left(\alpha_{2} A_{2}\right)$ and $m$ s $M$ i.e., $I$ is trivial.

If $C \leq I$, for $I$ an interval in $E$, then the image $L_{C}$ of $L$ under $q \times q$ : $I \times I \rightarrow(I / C) \times(I / C)$ is the relation on $J=I / C$ that is characterized, in terms of generalized elements $f, g: A \rightarrow J$, by $f \leq_{C} g$ iff there are an epi $\varepsilon: A_{1} \rightarrow A$ and maps $f_{1}, g_{1}: A_{1} \rightarrow I$ such that $q f_{1}=f \varepsilon, q g_{1}=g \varepsilon$ and $f_{1} \leq g_{1}$. Further, it is not hard to see that $L_{C}$ is reflexive, that $L_{C} \cup L_{C}^{-1}=$ $J \times J$ (but is, in general, neither transitive nor antisymmetric) and that $q m$ $(q M)$ is the minimum (maximum) element of $J$ (but they need not be disjoint). Thus $\left(I / C, L_{C}, q m, q M\right)$ is generally not an interval but under appropriate conditions on $C$ it is. $C$ is said to be convex in $I$ if, for each triple $f, g, h: A \rightarrow I$, if $f \leq g \leq h$ and $f, h$ each factors through $C$ then so does $g$. Denoting the image of $X \rightarrow 1$ by $|X|$ we have:

4.2 LemMA. If $C$ is a convex subobject of an interval I in a topos $E$ for which $|m \cap C| \cap|M \cap C|=0$ then $I / C$ is an interval in $E$. 
Proof. It suffices to prove that $\leq_{C}$ is transitive and antisymmetric and $q m \cap q M=0$. To show that $f \leq_{C} g$ and $g \leq_{C} h$ imply $f \leq_{C} h$, it is sufficient to construct an epi $\varepsilon^{\prime}: A^{\prime} \rightarrow A$ and maps $f^{\prime}, h^{\prime}: A^{\prime} \rightarrow I$ such that $f^{\prime} \leq h^{\prime}, q f^{\prime}=f \varepsilon^{\prime}$ and $q h^{\prime}=h \varepsilon^{\prime}$. To this end note that if $f \leq_{C} g$ and $g \leq_{C} h$ then (using a common pullback if necessary) there are an epi $\varepsilon: A_{1} \rightarrow A$ and maps $f_{1}, g_{1}, g_{1}^{\prime}, h_{1}: A_{1} \rightarrow I$ such that $f_{1} \leq g_{1}, g_{1}^{\prime} \leq h_{1}, q f_{1}=f \varepsilon$, $q g_{1}=g \varepsilon=q g_{1}^{\prime}$, and $q h_{1}=h \varepsilon$. Further, by comparability, there is an epi $\alpha=\alpha_{1}+\alpha_{2}: A_{11} \amalg A_{12} \rightarrow A_{1}$ with $f_{1} \alpha_{1} \leq h_{1} \alpha_{1}$ and $h_{1} \alpha_{2} \leq f_{1} \alpha_{2}$ and, since $q g_{1} \alpha_{2}=q g_{1}^{\prime} \alpha_{2}$ there is an epi $\beta=\beta_{1}+\beta_{2}: B_{1} \mathrm{II} B_{2} \rightarrow A_{12}$ for which $g_{1} \alpha_{2} \beta_{1}$ and $g_{1}^{\prime} \alpha_{2} \beta_{1}$ each factors through $C$ and $g_{1} \alpha_{2} \beta_{2}=g_{1}^{\prime} \alpha_{2} \beta_{2}$. Hence it follows from $g_{1}^{\prime} \alpha_{2} \beta_{l} \leq h_{1} \alpha_{2} \beta_{l} \leq f_{1} \alpha_{2} \beta_{i} \leq g_{1} \alpha_{2} \beta_{i}$ and the convexity of $C$, for $i=1$, and the antisymmetry of $\leq$, for $i=2$, that

$$
q g_{1}^{\prime} \alpha_{2} \beta_{1}=q h_{1} \alpha_{2} \beta_{1}=q f_{1} \alpha_{2} \beta_{1} \text { and } h_{1} \alpha_{2} \beta_{2}=f_{1} \alpha_{2} \beta_{2}
$$

respectively. Clearly the maps $f^{\prime}=f_{1} \alpha_{1}+g_{1}^{\prime} \alpha_{2} \beta_{1}+f_{1} \alpha_{2} \beta_{2}\left(h^{\prime}=h_{1} \alpha_{1}+\right.$ $\left.g_{1}^{\prime} \alpha_{2} \beta_{1}+h_{1} \alpha_{2} \beta_{2}\right): A^{\prime}=A_{11} \amalg B_{1} \amalg B_{2} \rightarrow I$ satisfy $f^{\prime} \leq h^{\prime}$ and $q f^{\prime}=f \varepsilon^{\prime}, q h^{\prime}$ $=h \varepsilon^{\prime}$ for the epi $\varepsilon^{\prime}=\varepsilon \alpha(\operatorname{id} \amalg \beta): A^{\prime} \rightarrow A_{11} \amalg A_{12} \rightarrow A_{1} \rightarrow A$. Thus transitivity is proved. To show antisymmetry note that $f \leq_{C} g$ and $g \leq_{C} f$ imply there are an epi $\varepsilon: A_{1} \rightarrow A$ and maps $f_{1}, g_{1}, g_{1}^{\prime}, f_{1}^{\prime}: A_{1} \rightarrow I$ such that $f \varepsilon=q f_{1}=q f_{1}^{\prime}, q \varepsilon=q g_{1}=g q_{1}^{\prime}$ and $f_{1} \leq g_{1}, g_{1}^{\prime} \leq f_{1}^{\prime}$. By comparability there is an epi $\alpha=\alpha_{1}+\alpha_{2}: A_{11} \amalg A_{12} \rightarrow I$ so that $f_{1} \alpha_{1} \leq g_{1}^{\prime} \alpha_{1}$ and $g_{1}^{\prime} \alpha_{2} \leq f_{1} \alpha_{2}$. Since $q f_{1} \alpha_{1}=q f_{1}^{\prime} \alpha_{1}$ there is an epi $\beta=\beta_{1}+\beta_{2}: B_{1} \amalg B_{2} \rightarrow A_{11}$ so that $f_{1} \alpha_{1} \beta_{1}$ and $f_{1}^{\prime} \alpha_{1} \beta_{1}$ each factors through $C$ and $f_{1} \alpha_{1} \beta_{2}=f_{1}^{\prime} \alpha_{1} \beta_{2}$. It then follows from $f_{1} \alpha_{1} \beta_{i} \leq g_{1}^{\prime} \alpha_{1} \beta_{i} \leq f_{1}^{\prime} \alpha_{1} \beta_{i}$ and the convexity of $C$, for $i=1$, and the antisymmetry of $\leq$, for $i=2$, that

$$
q f_{1} \alpha_{1} \beta_{1}=q g_{1}^{\prime} \alpha_{1} \beta_{1} \text { and } q f_{1} \alpha_{1} \beta_{2}=q g_{1}^{\prime} \alpha_{1} \beta_{2} .
$$

Since $\beta$ is epi we have that $q f_{1} \alpha_{1}=q g_{1}^{\prime} \alpha_{1}$, i.e., $f \varepsilon \alpha_{1}=g \varepsilon \alpha_{1}$. A similar argument shows that $f \varepsilon \alpha_{2}=g \varepsilon \alpha_{2}$ and thus, since both $\alpha$ and $\varepsilon$ are epi, $f=g$ and antisymmetry is proved. Finally note that, since 1 is terminal, the inclusion $j: q m \cap q M \rightarrow 1$ is just the equalizer of $q m$ and $q M$ and consequently there is an epi $\alpha=\alpha_{1}+\alpha_{2}: A_{1} \amalg \alpha_{2} \rightarrow q m \cap q M$ such that $m j \alpha_{1}$ and $M j \alpha_{1}$ each factors through $C$ and $m j \alpha_{2}=M j \alpha_{2}$. The first (resp. 2nd) condition implies that $\alpha_{1} A_{1}$ (resp. $\left.\alpha_{2} A_{2}\right)$ is a subobject of $|m \cap C|$ $\cap|M \cap C|=0$ (resp. of $m \cap M=0$ ) and thus, since $\alpha$ is epi, $q m \cap q M$ $=0$. This proves the lemma.

The proof of 1.3 is based on:

4.3 LEMMA. For each interval $I$ in a topos $E$ there is an irreducible interval $J$ in $E$ together with an endpoint preserving epi $I \rightarrow J$. 
Proof. If $C$ is either the subobject $\neg m$ or $\neg M$ in any interval $I$, then clearly $C$ is convex and $|m \cap C| \cap|M \cap C|=0$. Thus there is an endpoint preserving epi $q: I \rightarrow I / C$ to the interval, by $4.2, I / C$. Now the interval $I_{1}=\left(I /(\neg m), \quad m_{1}=q(m), \quad M_{1}=q(M)\right)$ satisfies $\neg m_{1}=M_{1}$ since, by 3.1 (d) with $A=m, \neg q(m)=q(\neg m)$, and, by 3.1(c) and the fact that $M \leq \neg m, q(\neg m)=q(M)$. Similarly, the interval $I_{2}=\left(I_{1} /\left(\neg M_{1}\right)\right.$, $\left.m_{2}=q\left(m_{1}\right), M_{2}=q\left(M_{1}\right)\right)$ satisfies $\neg M_{2}=m_{2}$ and $\neg m_{2}=M_{2}$; the latter equality since, by $3.1(\mathrm{c})$ and $m_{1} \leq \neg M_{1}$,

$$
\neg m_{2}=\neg q\left(m_{1}\right)=\neg q\left(\neg M_{1}\right),
$$

and by $3.1(\mathrm{a})$ and $\neg \neg M_{1}=\neg \neg \neg m_{1}=\neg m_{1}=M_{1}$,

$$
\neg q\left(\neg M_{1}\right)=q\left(\neg \neg M_{1}\right)=M_{2} .
$$

Thus $I_{2}$ is an irreducible interval and the composition $I \rightarrow I_{1} \rightarrow I_{2}=J$ is the desired epi.

4.4 Proof of 1.3. To see that (2), (3) each imply (1) note that each of these conditions implies, by 1.2 , that all irreducible intervals in $E$ are trivial. The result now follows from 4.3 since an interval $I$ is trivial iff there is an endpoint (i.e., $m, M$ ) preserving epi $I \rightarrow$ [2], where [2] $=1 \amalg 1$ is the (essentially unique) irreducible, trivial interval $(m, M$ are the canonical injections, 1 is the terminal object of $E$ ).

5. Intervals from subobjects. In this section we construct a special class of intervals that can be used to detect regular subobjects of 1 with complements. The main result, and the basis of the proof of 1.4 is:

5.1 LEMMA. For each subobject $U$ of 1 in a topos $E$ there is an interval $I(U)$ in E such that:

(1) $I(U)$ is irreducible iff $U$ is regular, and

(2) $I(U)$ is trivial iff $\neg \neg U$ has a complement. Thus all regular subobjects of 1 have complements if all irreducible intervals in $E$ are trivial.

Proof. Clearly the functor $S \mapsto \amalg_{S} 1$ from the category of finite sets to the topos $E$ maps linearly ordered sets to linearly ordered objects of $E$. In particular, the ordinal $\{0,1,2\}$ defines an interval [3] $=1 \amalg 1 \amalg 1$ in $E$, in which $m=i^{0}, M=i^{2}$, where $i^{p}: 1 \rightarrow[3], p=0,1,2$, are the canonical injections, and, in terms of generalized elements $f, g: A \rightarrow[3], f \leq g$ iff, as subobjects of $A, g^{*}(m) \leq f^{*}(m)$ and $f^{*}(M) \leq g^{*}(M)$. For a subobject $U \rightarrow 1$, the obvious injection $C=U$ IIIII $U \rightarrow$ [3] defines $C$ as a convex 
subobject. To see this note that a map $f: A \rightarrow[3]$ factors through $C$ iff $\left|f^{*}(m)\right| \leq U$ and $\left|f^{*}(M)\right| \leq \neg U$. Thus, if $f, g, h: A \rightarrow$ [3] with $f \leq g \leq h$ and $f, g$ each factors through $C$ then $g^{*}(m) \leq f^{*}(M),\left|f^{*}(m)\right| \leq U$, $g^{*}(M) \leq h^{*}(M)$, and $\left|h^{*}(M)\right| \leq \neg U$; consequently $\left|g^{*}(m)\right| \leq U$, $\left|g^{*}(M)\right| \leq \neg U$ and $g$ factors through $C$. Further, it is trivial to see that $|m \cap C|=U$ and $|M \cap C|=\neg U$. Hence, by $4.2, I(U)=[3] / C$ is an interval in $E$ with $m=q\left(i^{0}\right)$ and $M=q\left(i^{2}\right)$ for $q:$ [3] $\rightarrow I(U)$ the defining epi. A direct calculation shows that, as subobjects of [3], $q^{*}(m)=1 \amalg U \amalg 0$ and $q^{*}(M)=0 \amalg \neg U \amalg 1$. Thus $\neg q^{*}(m)=q^{*}(M)$ and, since $q$ is epi,

$$
\neg m=q q^{*}(\neg m)=q\left(\neg q^{*}(m)\right)=q q^{*}(M)=M .
$$

Further, $\neg \neg U=U$ iff $\neg q^{*}(M)=q^{*}(m)$ iff $\neg M=m$. This shows (1). For (2) note that if $\neg \neg U \mathrm{II} \neg U=1$ then

$$
\begin{aligned}
C & =\left(\left(C_{1}=U \amalg \neg \neg U \amalg 0\right) \amalg\left(C_{2}=0 \amalg \neg U \amalg \neg U\right)\right) \\
& \rightarrow\left(\left(I_{1}=1 \amalg \neg \neg U \amalg 0\right) \amalg\left(I_{2}=0 \amalg \neg U \amalg 1\right)\right)=[3]
\end{aligned}
$$

and $I(U) \simeq I_{1} / C_{1} \amalg I_{2} / C_{2}$ renders $I(U)$ trivial.

5.2 Proof of 1.4. Part (a) is a direct consequence of 5.1. For (b) note that since the endpoints of an irreducible interval are obviously regular, they are mutually complementary and thus all irreducible intervals in $E$ are trivial. The result now follows from 4.3 as in the proof of 1.3. For (c) it is sufficient to show that, for any regular subobject $A$ of $X$ in $E$, the obvious mono $A \amalg_{\neg} A \rightarrow X$ is epi (and thus an isomorphism) which, under the $S G$ assumption, can be accomplished by showing that each partial map $1 \rightarrow X$ factors through it. However, the pullback of $A$ along any such partial map $U \rightarrow X$ defines a regular subobject $U_{1}$ of $U$, which satisfies, as a subobject of $1, \neg \neg U_{1} \amalg \neg U_{1}=1$ and consequently $\left(\neg \neg U_{1} \cap U\right) \amalg\left(\neg U_{1} \cap\right.$ $U)=U$. But since $U_{1}$ is regular in $U, U_{1}=\neg \neg U_{1} \cap U$, and since $U_{1}$ is the pullback of $A,\left(\neg U_{1} \cap U\right)$ is the pullback of $\neg A$ and thus

$$
U=U_{1} \amalg\left(\neg U_{1} \cap U\right) \rightarrow A \amalg \neg A \rightarrow X
$$

gives the desired factorization.

We conclude with the observation that if $G \rightarrow 1$ is the generic subobject (i.e., classified by the generic element $1 \rightarrow \Omega^{*}$, p. 39 [2]) in $E / \Omega$ then the interval $I(G)=(I \rightarrow \Omega)$ in $E / \Omega$ is universal for the intervals $I(U)$ in the sense that $I(U) \simeq f^{*}(I)$, where $f: 1 \rightarrow \Omega$ is the classifying map of $U$. Moreover, the restriction of $I(G)$ to $\Omega_{\neg \neg}$ is the universal interval for the irreducible $I(U)$ 's. 


\section{REFERENCES}

[1] O. Frink, Pseudo-complements in semi-lattices, Duke Math J., 29 (1962), 505-514.

[2] P. T. Johnstone, Topos Theory, L.M.S. Math Monograph No. 10 (1977) Academic Press.

[3] Conditions related to DeMorgan's Law, Applications of Sheaves, Springer Lecture Notes in Math., 753 (1979), 479-491.

[4] Another condition equivalent to DeMorgan's Law, Communications in Algebra, 7 (12) (1979), 1309-1312.

[5] _ On a topological topos, Proc. London Math. Soc., (3) 38 (1979), 237-271.

[6] - The Gleason cover of a topos, I, J. Pure Appl. Algebra, 19 (1980), 171-192.

[7] _ The Gleason cover of a topos, II, J. Pure Appl. Algebra, 22 (1981), 229-247.

Received December 9, 1981 and in revised form June 15, 1982.

UNIVERSITY OF MIAMI

Coral Gables, FL 33124 


\section{PACIFIC JOURNAL OF MATHEMATICS \\ EDITORS}

DONALD BABBITT (Managing Editor)

University of California

Los Angeles, CA 90024

Hugo RossI

University of Utah

Salt Lake City, UT 84112

C. C. MOOre and ARThur OGus

University of California

Berkeley, CA 94720
J. Dugundi

Department of Mathematics

University of Southern California

Los Angeles, CA 90089-1113

R. FINN and H. SAMELSON

Stanford University

Stanford, CA 94305

ASSOCIATE EDITORS

$\begin{array}{lllll}\text { R. Arens } & \text { E. F. Beckenbach } & \text { B. H. Neumann } & \text { F. Wolf } & \text { K. Yoshida }\end{array}$ $(1906-1982)$

\section{SUPPORTING INSTITUTIONS}

UNIVERSITY OF ARIZONA

UNIVERSITY OF BRITISH COLUMBIA

CALIFORNIA INSTITUTE OF TECHNOLOGY

UNIVERSITY OF CALIFORNIA

MONTANA STATE UNIVERSITY

UNIVERSITY OF NEVADA, RENO

NEW MEXICO STATE UNIVERSITY

OREGON STATE UNIVERSITY
UNIVERSITY OF OREGON

UNIVERSITY OF SOUTHERN CALIFORNIA

STANFORD UNIVERSITY

UNIVERSITY OF HAWAII

UNIVERSITY OF TOKYO

UNIVERSITY OF UTAH

WASHINGTON STATE UNIVERSITY

UNIVERSITY OF WASHINGTON 


\section{Pacific Journal of Mathematics}

\section{Vol. 110, No. $1 \quad$ September, 1984}

Wojciech Abramczuk, A class of surjective convolution operators $\ldots \ldots \ldots \ldots 1$

K. Adachi, Extending bounded holomorphic functions from certain

subvarieties of a weakly pseudoconvex domain $\ldots \ldots \ldots \ldots \ldots \ldots$

Malvina Florica Baica, An algorithm in a complex field and its application

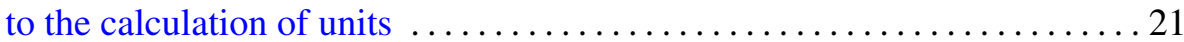

Giuliana Bianchi and Robert Cori, Colorings of hypermaps and a

conjecture of Brenner and Lyndon $\ldots \ldots \ldots \ldots \ldots \ldots \ldots \ldots \ldots \ldots \ldots \ldots$

Ronald James Evans, Determinations of Jacobsthal sums . . .......... 49

Leslie Foged, Characterizations of $\aleph$-spaces .................... 59

Nassif A. Ghoussoub and Paulette Saab, Weak compactness in spaces of

Bochner integrable functions and the Radon-Nikodým property . . . . . . 65

J. Gómez Gil, On local convexity of bounded weak topologies on Banach

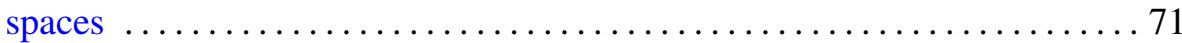

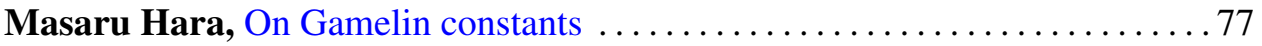

Wilfried Hauenschild, Eberhard Kaniuth and Ajay Kumar, Harmonic

analysis on central hypergroups and induced representations $\ldots \ldots \ldots 83$

Eugenio Hernandez, An interpolation theorem for analytic families of

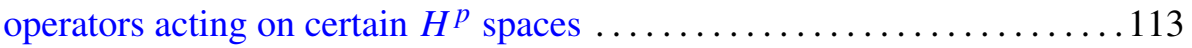

Thomas Alan Keagy, On "Tauberian theorems via block-dominated

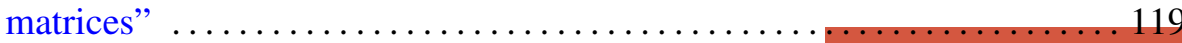

Thomas Landes, Permanence properties of normal structure $\ldots \ldots \ldots \ldots \ldots 125$

Daniel Henry Luecking, Closed ranged restriction operators on weighted

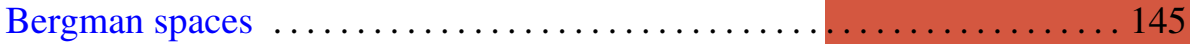

Albert Thomas Lundell, The $p$-equivalence of $\mathrm{SO}(2 n+1)$ and $\mathrm{Sp}(n) \ldots \ldots 161$

Mark D. Meyerson, Remarks on Fenn's "the table theorem" and Zaks' "the chair theorem" ..................................... 167

Marvin Victor Mielke, Homotopically trivial toposes . . . . . . . . . . 171

Gerard J. Murphy, Hyperinvariant subspaces and the topology on Lat A . . 183

Subhashis Nag, On the holomorphy of maps from a complex to a real manifold

Edgar Milan Palmer and Robert William Robinson, Enumeration of self-dual configurations ................................. 203

John J. Walsh and David Clifford Wilson, Continuous decompositions

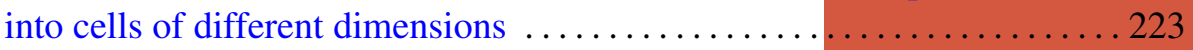

Walter John Whiteley, Infinitesimal motions of a bipartite framework .....233 